\title{
Effect of Turbulence on Flame Propagation in Cornstarch Dust-Air Mixtures
}

\section{Shuangfeng WANG ${ }^{\mathbf{1}} \quad$ Yikang PU $^{\mathbf{1}}$ Fu JIA $^{\mathbf{1}} \quad$ Artur GUTKOWSKI $^{\mathbf{2}}$}

1. National Microgravity Laboratory, Institute of Mechanics, Chinese Academy of Sciences, Beijing 100080, China

2. Department of Heat Technology and Refrigeration, Technical University of Lodz, Stefanowskiego 1/15, 90-924 Lodz, Poland

\begin{abstract}
Following the quantitative determination of dust cloud parameters, this study investigated the flame propagation through cornstarch dust clouds in a vertical duct of $780 \mathrm{~mm}$ height and $160 \times 160 \mathrm{~mm}$ square cross section, and gave particular attention to the effect of turbulence on flame characteristics. The turbulence induced by dust dispersion process was measured using a particle image velocimetry (PIV) system. Upward propagating dust flames were visualized with direct light and shadow photography. The results show that a critical value of the turbulence intensity can be specified below which laminar flame propagation would be established. This transition condition is about $10 \mathrm{~cm} / \mathrm{s}$. The measured propagation speed of laminar flames appears to be in the range of $0.45-0.56 \mathrm{~m} / \mathrm{s}$, consistent with the measurements reported in the literature. For the present experimental conditions, the flame speed is little sensitive to the variations in dust concentration. Some information on the flame structure was revealed from the shadow records, showing the typical heterogeneous feature of dust combustion process.
\end{abstract}

Keywords: dust flame, flame propagation, dust dispersion process, turbulence, cornstarch. CLC number: V231.2+6 Document code: A Article ID: 1003-2169(2006)02-0186-07

\section{Introduction}

Combustion of dust clouds in air is of great practical importance due to safety problems related to dust explosion hazards, as well as to utilization of solid fuels. Numerous experimental and theoretical studies on the characteristics of dust combustion have been conducted $^{[1 \sim 7]}$. Nevertheless, knowledge on the fundamental mechanism of flame propagation in dust-air mixtures is still far from sufficient. Among several great obstacles to dust combustion research, the most fundamental one may be the understanding and dealing with the influence of turbulence on flame propagation.

From a practical standpoint, turbulent flame propagation has been a central problem in combustion science since almost all combustion processes take place under somewhat turbulent conditions. For dust combustion, however, the influence of turbulence is also an inherent part of the experimental study. In the experiments, dust particles must be suspended in the air to form a combustible dust cloud. This prerequisite is accomplished

Received Nov. 10, 2005

Shuangfeng WANG: Associate Professor by a process called dust dispersion. Various types of dispersion process ${ }^{[5]}$ used for dust combustion research will generate turbulence in the test vessel, which, on the other hand, is proven necessary to maintain dust in suspension. Therefore some degree of turbulence is always present in the dust cloud before ignition. The turbulence structures may vary from one apparatus to the next, depending on factors such as the dispersion method and vessel geometry. It appears that the basic question is perhaps not merely how to produce an experimental dust cloud, but rather whether a definition of the cloud states can be given. Provided the quantitative information on the state of a dust cloud (dust concentration, dynamic state, etc.) is obtained, one can correlate the observed flame propagation behaviors with this state, and correlate experimental data from different workers who have used different apparatus. However, comparatively little quantitative knowledge exists about dust dispersion processes and the resulting states of the cloud, even after Eckhoff ${ }^{[8]}$ proposed that experimental investigation into such information should be encouraged. Herein lies an 


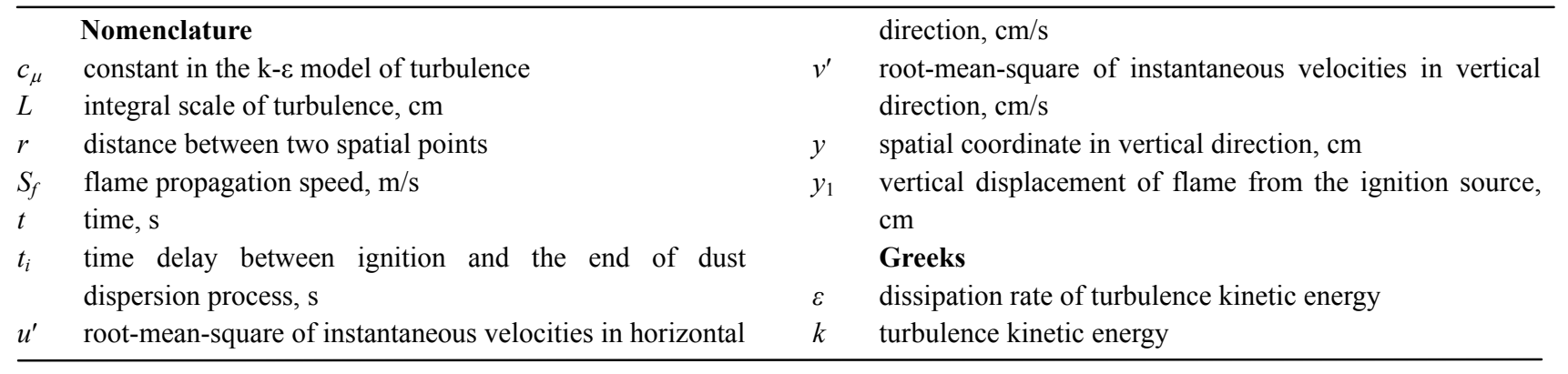

important reason why the limited experimental results of dust flame propagation are often apparatus dependent and contradict each other in literature.

In view of this, the goal of the present study was to determine quantitatively the states of dust cloud, and observe flame propagation in this cloud. With an introduced dispersion mechanism, the features of dispersion-induced turbulence, dust concentrations and their variations with time were measured in a vertical duct. The upward cornstarch dust flame, which propagated from the open bottom to the closed top of the duct, was examined through photographic records. Based on the experimental results, the effects of turbulence and dust concentration on flame propagation were quantitatively discussed.

\section{Experimentation}

\section{Experimental apparatus and procedures}

A simplified schematic of the experimental system is shown in Fig.1. Its central part is a vertical duct of 780 $\mathrm{mm}$ height and $160 \times 160 \mathrm{~mm}$ square cross section, made of glass in order to obtain visualization of flames in combustion experiments and dust particles (illuminated by a laser light sheet) in dust dispersion experiments. The newly designed dispersion mechanism consists of a dust cloud generator and an airflow feeder with pressure controller. The generator, including a porous metallic plate (of $3 \mathrm{~mm}$ thickness, $20 \sim 50 \mu \mathrm{m}$ pore size) and an air chamber of the same cross section as the duct, is downward detachable from the duct at a given time by means of an electromagnet controlling system. The experimental procedure is as follows. Dust particles were evenly distributed on the fine porous plate at the bottom of the duct. With the condition of the duct top open, compressed air at appropriate rates was introduced through the porous plate producing small scale jets to disperse the dust particles. The dust suspension flows within the duct up to the top end. When the duct is completely filled with a dust cloud, a time controlling system simultaneously stops the airflow (the end of dust dispersion), separates the dust cloud generator from the bottom of the duct and closes the shutter at the top end. In order to examine the influence of dispersion-induced turbulence on flame propagation, the ignition time is delayed by $0.5 \sim 3.2 \mathrm{~s}$ from the end of dispersion (ignition delay time). After the delay, the dust-air mixture

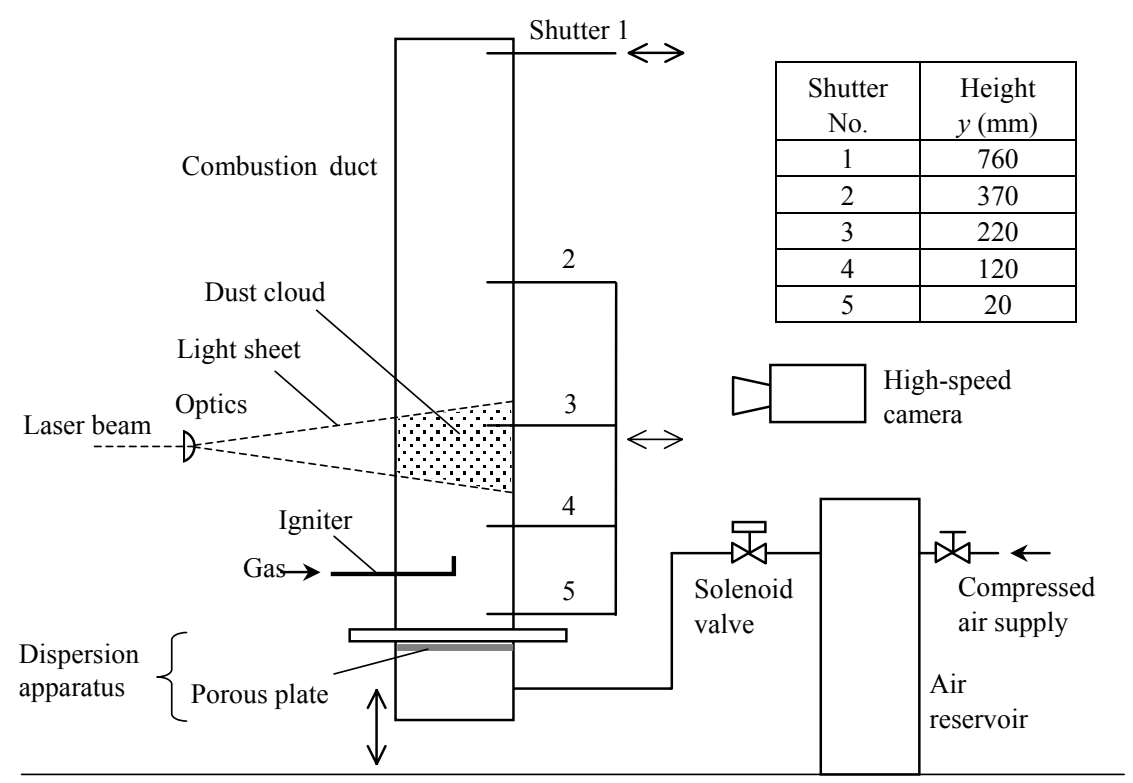

Fig.1 Experimental setup 
was ignited above the bottom end of the duct by a device resembling a cigarette lighter. A spark with very small energy ignited a small amount of butane-air mixture, and then the gas flame ignited the dust clouds. Thus, the dust flame propagates from the open end of the duct (at the bottom) up to the closed end (at the top).

Cornstarch $\left(\mathrm{C}_{6} \mathrm{H}_{10} \mathrm{O}_{5}\right)$ was used as the fuel. The particles were nearly spherical in shape with a mass mean diameter of $14 \mu \mathrm{m}$.

\section{Measurements}

Three sets of experiments were performed: the first two were devoted to the measurements of dispersioninduced turbulence and dust concentration (without ignition), respectively, and the third to flame propagation observation.

The determination of the feature of dispersioninduced flow was accomplished using a particle image velocimetry (PIV) measurement system. The flow was seeded by the cornstarch dust particles themselves, which were illuminated by a laser light sheet. The movement of dust particles was recorded by a high-speed camera at 100 frames/s, and was subsequently analyzed using a digital image processing system for the quantitative measurements of instantaneous particle velocities. The field of view in PIV measurements was $40 \times 30 \mathrm{~mm}$. Because of its strongly nonstationary nature, it is necessary to utilize the ensemble average method to determine statistical properties of the dispersion-induced turbulence ${ }^{[9,10]}$. More than 90 runs of dust dispersion experiment have been repeated. For each test, instantaneous velocity fields at different times relative to the end of dust dispersion were acquired by PIV. The ensemble statistics were constructed by averaging the instantaneous measurements at corresponding times and positions over the various tests.

For the dust concentration measurements, four other horizontal shutters were installed below the top one along the height of the duct. These shutters slid into the duct simultaneously at a certain time after the end of dispersion process, and divided the interior space into four sections. The mean concentration was evaluated by weighing dust particles settled on the shutters with overall accuracy of $0.01 \mathrm{~g}$. In this way, variation of dust concentration along with the duct and with time can be estimated.

The experimental apparatus was established so as to allow visualization of flames. Two kinds of photographic records were performed: self emitted light and shadow method records. The former was used to obtain the flame propagation over a distance of about $350 \mathrm{~mm}$. For the shadow observation, two opposite windows of optical glass (of $150 \mathrm{~mm}$ diameter and $12 \mathrm{~mm}$ thickness) were installed on the side walls of the duct. The windows were about $300 \mathrm{~mm}$ above the bottom of the duct. Fig.2 shows how the shadow system employs a $300 \mathrm{~mW}$ laser as light source to produce flame shadow. In both cases, the flame propagation was recorded by the high-speed camera with framing rate of 250 frames/s.

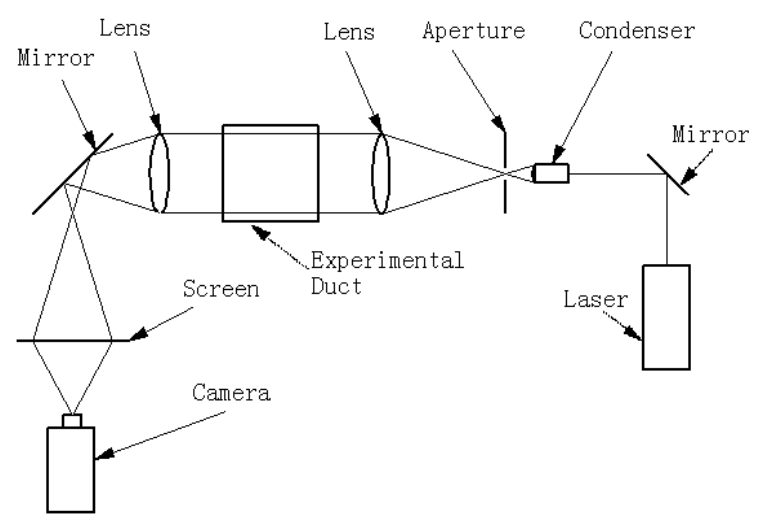

Fig.2 Schematic of the shadow system

\section{Results and Discussion}

\section{Dispersion-induced turbulence}

The turbulence intensity is expressed by the rootmean-square (RMS) of the instantaneous velocities in horizontal and vertical directions. The results of ensemble average process are given in Fig.3(a) as a function of time, where the data at three typical positions (the central point, lower left corner and upper right corner of the observation window) are displayed and collapse well on one curve. In fact, the RMS instantaneous velocities are also evaluated for many other positions. They appear very similar in values to those illustrated in Fig.3(a), and show the same tendency to decay with time. The timedependent turbulence intensity (in $\mathrm{cm} / \mathrm{s}$ ) in horizontal direction, $u^{\prime}$, and in vertical direction, $v^{\prime}$, can be matched by the following expressions:

$$
\begin{aligned}
& u^{\prime}=14.17(1+t)^{-0.794} \\
& v^{\prime}=18.87(1+t)^{-0.715}
\end{aligned}
$$

In Fig.3(a), noteworthy is that the maximum value of RMS velocity is less than $20 \mathrm{~cm} / \mathrm{s}$, while for previous dispersion systems this value may reach as high as several meters per second ${ }^{[10,11]}$. The much lower level of velocity fluctuation obtained with the present dispersion mechanism is significant to laminar dust combustion studies. The vertical RMS velocities are systematically higher than horizontal ones, which obviously reflects the effect of gravity-induced settlement of dust particles. Furthermore, the decay of the turbulence intensity is much slower than previous dispersion systems, implying that the dust suspension could be maintained for a longer period of time. 


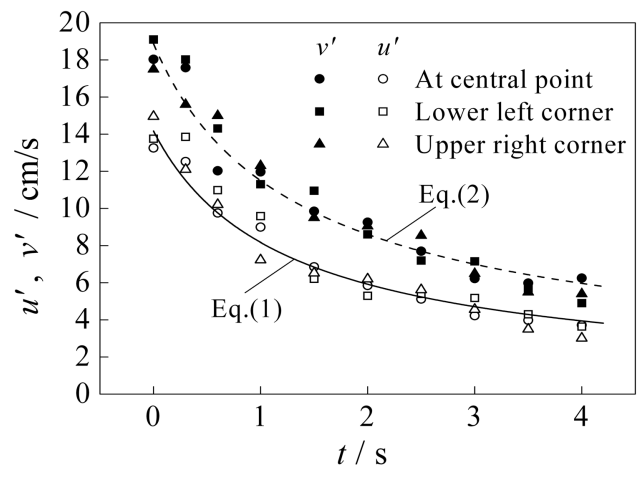

(a) Turbulence intensity

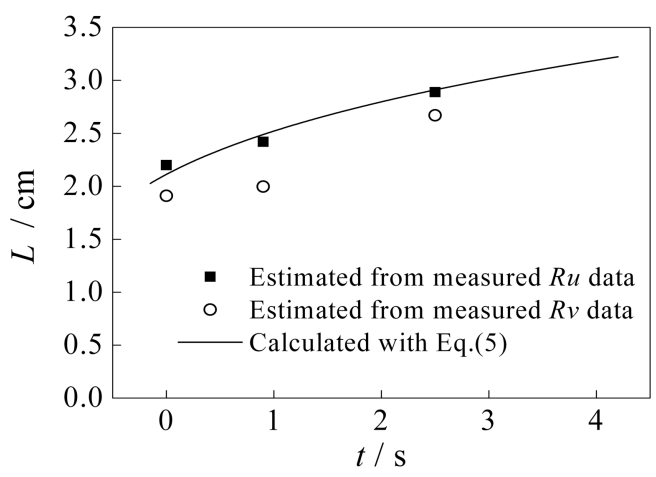

(b) Integral scale

Fig.3 Parameters of dispersion-induced turbulence as a function of time

On basis of the PIV measurements of instantaneous velocity field, the integral scale of dispersion-induced turbulence, $L$, is readily derived according to its definition $^{[9]}$ :

$$
L_{u}(t)=\int_{0}^{\infty} R_{u}(r, t) d r
$$

or

$$
L_{v}(t)=\int_{0}^{\infty} R_{v}(r, t) d r
$$

where $R_{u}$ and $R_{v}$ are the correlation coefficients of the horizontal and vertical instantaneous velocities, respectively, and $r$ is the distance between two points. For three instants $(t=0,0.9$ and $2.5 \mathrm{~s})$, the results are shown in Fig.3(b). It can be seen that the integral scale deduced from $R_{u}$ conforms that from $R_{v}$, although the former is a little higher. At the end of the dust dispersion process $(t=0)$, the turbulence has a length scale of about $2 \mathrm{~cm}$. The scale increased with time, and has a value of about $2.8 \mathrm{~cm}$ at $t=2.5 \mathrm{~s}$.

Alternatively, Tamanini and Chaffee ${ }^{[12]}$ described a method through which information on the integral scale of turbulence can be obtained. The method is based on the definition of $L$ as a function of the turbulence kinetic energy, $k$, and its dissipation, $\varepsilon$, given by:

$$
L=c_{\mu}^{3 / 4} \frac{k^{3 / 2}}{\varepsilon}
$$

In the present dispersion-induced turbulence field, it is reasonable to assume the RMS of two component velocities in horizontal directions to be equal, which yields the relationship $k=u^{\prime 2}+(1 / 2) v^{\prime 2}$. When Eqs. (1) and (2) are used, and the definition of $\varepsilon(=-d k / d t)$ is invoked, the integral length scale can be predicted by Eq. (5) for a value of the constant $c_{\mu}=0.09$, as normally used in the $\mathrm{k}-\varepsilon$ model of turbulence. The calculated variation of $L$ with time is also presented in Fig.3(b), and the good agreement in the comparison to the experimental measurements is demonstrated.

\section{Dust concentration}

The determination of dust concentrations was based on a set of five repeated tests, and the error span was within $15 \%$ of the averaged value. Shown in Fig. 4 is the time variation of the mean concentrations at four heights of the duct ( $y=70,170,295$ and $565 \mathrm{~mm}$ ). It is evident that the dust concentration decreases with time and also along the height of the duct. Over the typical propagation distance of dust flames observed in the experiments $(y=$ $100 \sim 450 \mathrm{~mm})$, the concentration difference can be $2 \sim 3$ times. Immediately after the end of dust dispersion process, dust concentrations decrease rather sharply because of the disappearance of dispersion airflow. From $t=0.5 \mathrm{~s}$, however, the variation becomes so gradual that when $t=2.8 \mathrm{~s}$ the dust concentration at $y=565 \mathrm{~mm}$ is still kept more than $90 \mathrm{~g} / \mathrm{m}^{3}$, well above the lean flammability limit of cornstarch dust-air mixtures (70 $\mathrm{g} / \mathrm{m}^{3}$, as reported by Proust and Veyssiere ${ }^{[3]}$ ).

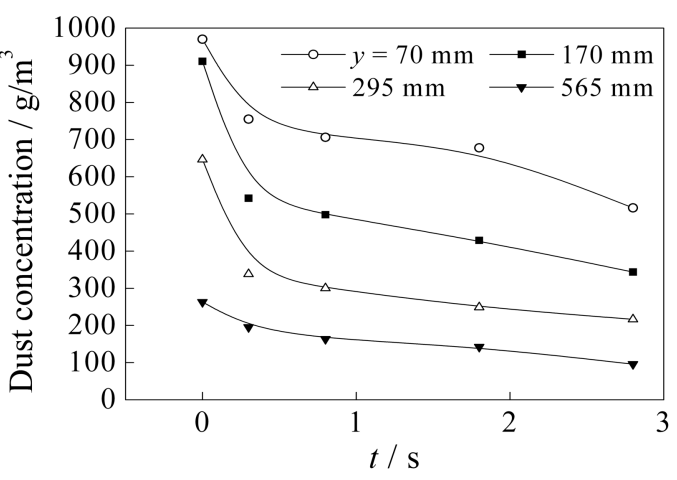

Fig.4 Mean dust concentration as a function of time at four heights of the duct

\section{Flame propagation in cornstarch dust clouds}

By choosing the ignition delay time, $t_{i}$, the flame propagates in cornstarch dust-air mixtures with different initial state in terms of turbulence level and dust 
concentration. In the experiments two kinds of flames can be observed according to the delay time: laminar and turbulent. The typical laminar flames have been observed for delay time greater than $1.4 \mathrm{~s}$, while for $t_{i}$ less than 1.1 $\mathrm{s}$ the experimental conditions give rise to turbulent type flames. Examples of a laminar flame and a turbulent flame are shown in Fig.5, which are recorded directly by the high-speed camera. As can be seen, the laminar flame front is almost smooth with a parabolic-like form, which is a typical feature of laminar propagating flames in a duct. The turbulent flame seems to be characterized mainly by an irregular front and a higher propagation speed. Additionally, the total range of the luminous reaction zone is wider than that for laminar flames.
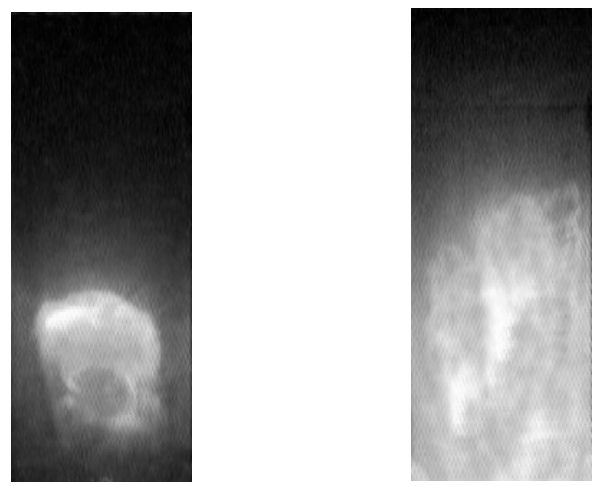

$\begin{array}{ll}\text { (a) a laminar flame, } t_{i}=1.4 \mathrm{~s} & \text { (b) a turbulent flame, } t_{i}=0.6 \mathrm{~s}\end{array}$

Fig.5 Photographs of typical flames propagating in cornstarch dust clouds
The shadow method can reveal more details of flame structure. As an illustration, sequences of shadow records of propagating laminar and turbulent flames are reproduced in Figs.6(a) and (b). In these images, a narrow dark zone can be seen clearly ahead of the flame front, which roughly represents the preheat zone typical of the volatile type dust flames. The visualization of turbulent flames shows a very irregular distribution of regions with local reactions behind the flame front. As the time goes, the main flame front is notably stretched in vertical direction so that it is separated from the following flames by increasing distance. This means that these flames propagate with different speeds, and the highest speed is for the main flame. For laminar dust combustion, a few separated flames are also observed behind the main flame front, although they are much less visible than in turbulent flames because of the limited extent. It is evident from Fig.6 that the dust flames have complex structure in comparison with those in homogeneous gaseous mixtures. The multi-layer structure of dust flames suggests that the combustion takes place not ideally at a single flame front, instead it occurs at a cluster of surfaces where the gaseous decomposition of dust particles is available. Such information help to explain the significant effects of dust particle distribution and turbulence on the combustion process.

To characterize these different flame propagation regimes in the duct, an important parameter used is the flame propagation speed. Its value can be derived from the photographic records through an image analysis
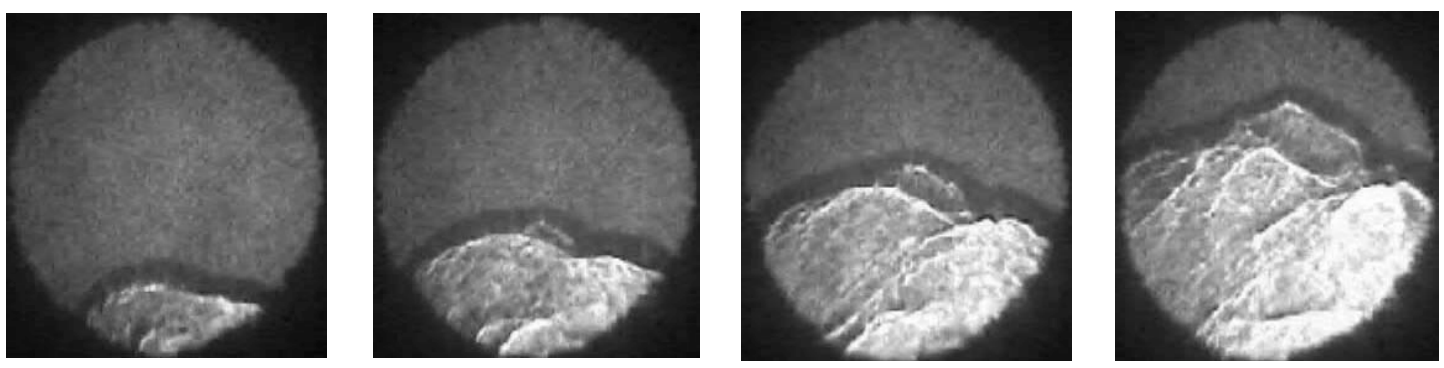

(a) A laminar flame, time interval between pictures $0.012 \mathrm{~s}$
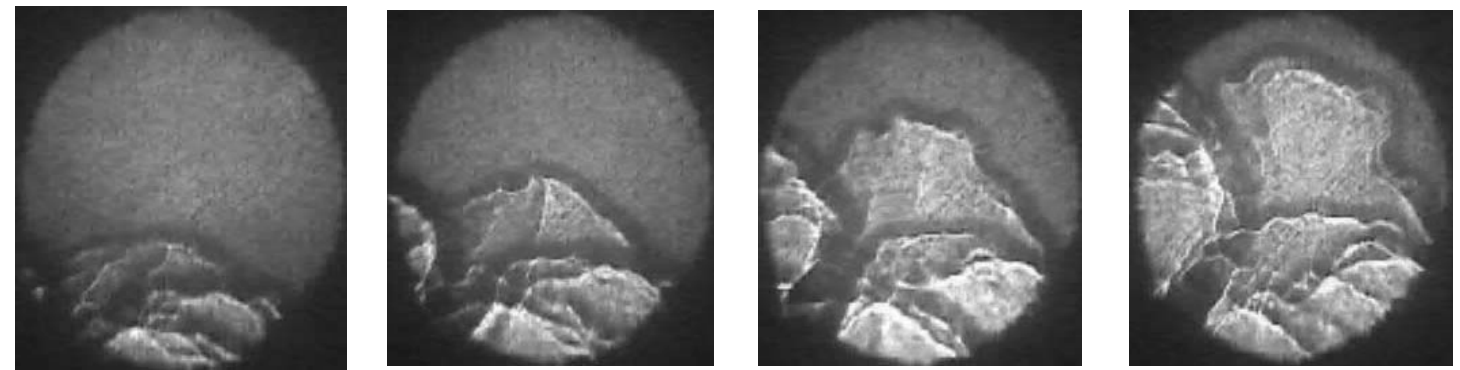

(b) A turbulent flame, time interval between pictures $0.012 \mathrm{~s}$

Fig.6 Shadow photographic records of cornstarch dust flame propagation 
process to identify the flame leading edge and then calculating the vertical displacement of the flame. Fig.7 shows the measured flame displacement (from the ignition source), $y_{1}$, as a function of time for $t_{i}=0.6,1.4$, 1.8 and 3.2 s. Obviously, a linear relationship exists between $y_{1}$ and $t$ under all conditions of ignition delay time, indicating that for a given delay time the flame propagates with constant speed in the duct. Thus the average slope of the flame coordinates in Fig.7 represents the propagation speed, $S_{f}$. Results of such measurements are reported in Fig. 8 as a function of the ignition delay time. It is interestingly noticed that the variance of $S_{f}$ with $t_{i}$ has two regimes, and the transition occurs approximately at $t_{i}=1.1 \mathrm{~s}$. In the left regime (where turbulent propagating flames were observed), the flame speed is as high as the order of $0.9 \mathrm{~m} / \mathrm{s}$. In the right propagation regime (laminar flames), the flame speed is much lower $\left(S_{f}=0.45 \sim 0.56 \mathrm{~m} / \mathrm{s}\right)$ and does not vary much with the ignition delay time. Both for turbulent flames and for laminar flames, the propagation speed is quite close to what reported by Proust and Veyssiere ${ }^{[3]}$ (for turbulent flames $S_{f} \approx 0.8 \mathrm{~m} / \mathrm{s}$, for laminar flames $S_{f}=0.45 \sim 0.63 \mathrm{~m} / \mathrm{s}$ ), although these authors did not quantitatively assess the turbulence parameters in experimental dust clouds and determined the state (laminar or turbulent) of the clouds simply by observation.

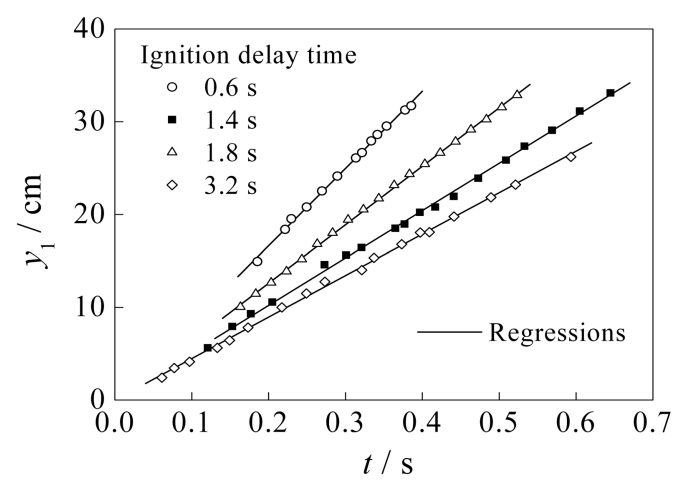

Fig.7 Displacement of flame front as a function of time

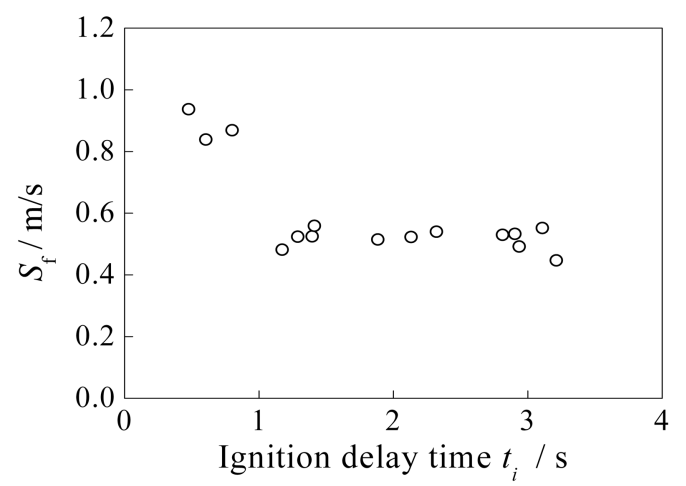

Fig.8 Variation of flame propagation speed with ignition delay time
As mentioned above, the dust concentration decreases considerably along the height of the duct, and yet the cornstarch dust flame propagates upwards at a constant speed for any given ignition delay time. This observation may suggest that the dust concentration has little influence on flame propagation speed. For laminar cornstarch dust flames, Proust and Veyssiere ${ }^{[3]}$ have also found that the propagation is only slightly affected by dust concentration in the $75 \sim 580 \mathrm{~g} / \mathrm{m}^{3}$ range. Furthermore, they deduced the laminar burning velocity from $S_{f}$ measurements and photographically estimated flame surface areas, and found that its value does not vary much with dust concentration. The present experimental results are in agreement with the finding of Proust and Veyssiere, showing even less effect of dust concentration. It is clear, however, that more research is needed to understand the real role of dust concentration in flame propagation.

The observation of flame propagation regimes and the flame speed data in Fig. 8 have suggested that the ignition delay time determines the features of cornstarch dust flames propagating in the duct. Because the dust concentration is proven to be little influential factor in flame propagation, the change in flame behaviors with increasing delay time could be attributable to the decrease of turbulence intensity, i.e. the turbulence intensity can be regarded as an independent parameter to examine its influence. As a quantitative measure, one can decide that the transition from turbulent to laminar flames occurs close to the ignition delay time $1.1 \mathrm{~s}$. This critical condition, from the measurements of turbulence intensity, corresponds to a turbulence intensity of $8 \mathrm{~cm} / \mathrm{s}$ in horizontal direction, and $11 \mathrm{~cm} / \mathrm{s}$ in vertical direction. Since the ideal static (or laminar) and uniform dust cloud is impossible to realize under normal gravity conditions ${ }^{[4,5,13]}$, such a critical value of the turbulence level can serve for practical condition under which the laminar dust flame would be obtained. Certainly, the specific value of 10 $\mathrm{cm} / \mathrm{s}$ is a preliminary one, and should be justified through further investigation.

\section{Summary}

The experimental study was conducted to elucidate the effects of turbulence on the flame propagation in cornstarch dust clouds. For this purpose, an improved dust dispersion mechanism was designed, and the resulting states of dust clouds were determined in a vertical duct of $780 \mathrm{~mm}$ height and $160 \times 160 \mathrm{~mm}$ square cross section. The feature of dispersion-induced turbulence, including turbulence intensity, integral scale and their variations with time was determined by means of a PIV measurement system and ensemble average method. Dust concentrations were measured by direct weighing 
method. The results show that the turbulence level induced by the dispersion process is less than $20 \mathrm{~cm} / \mathrm{s}$, much lower than those obtained with previous dispersion system, and the decay of turbulence is slow. The integral scale of the turbulence increases with time from 2 to 3 $\mathrm{cm}$ during about $3 \mathrm{~s}$. The decrease of dust suspension with time is also slow, indicating that dust suspensions in air could be maintained during a relatively long period.

Based upon the dust dispersion apparatus, the upward flame propagation was established in the duct, with the top end closed and the bottom end open. The flames were visualized with a high-speed camera and with the shadow method. With the increase of ignition delay time, two different kinds of flame were observed: turbulent and laminar flames. The transition occurs when the ignition delay time is about $1.1 \mathrm{~s}$, corresponding to a turbulence level of $10 \mathrm{~cm} / \mathrm{s}$. Both kinds of flames propagate with steady speed in the duct. The propagation speed of turbulent flames is in the order of $0.9 \mathrm{~m} / \mathrm{s}$. For laminar flames, the propagation speed is in the range of $0.45 \sim 0.56 \mathrm{~m} / \mathrm{s}$, and does not vary much with the ignition delay time. Under the present experimental conditions, it was found that the flame propagation velocity is a very weak function of dust concentration in cornstarch dust clouds.

The observation of shadow images showed that the flame structure contains preheat and combustion zones typical of the volatile type dust flames. A very irregular distribution of separated flames was observed to occur behind the flame front, especially in turbulent combustion zones. Such heterogeneous feature indicated the importance of the state of dust clouds.

\section{Acknowledgements}

The work reported here was sponsored by the Knowledge Innovation Major Project of Chinese Academy of Sciences (CAS) under grant number KJCX2-SW-L05. The authors wish to thank Prof. J. Jarosinski, Technical University of Lodz, Poland, for helpful discussions and criticism. One of the authors, A. Gutkowski, was financially supported by the Marie Curie Transfer of Knowledge Project (grant No. ECHIRA NR 509849) during his stay at the National Microgravity Laboratory, CAS.

\section{References}

[1] Krazinski, J L, Buckius, R O, Krier, H. Coal Dust Flames: a Review and Development of a Model for
Flame Propagation. Prog. Energy Combust. Sci., 1979, 5(1): $31-71$

[2] Jarosinski, J, Lee, J H, Knystautas, R, et al. Quenching Distance of Self-propagation Dust-air flames. In: Proceedings of 21st Symposium (International) on Combustion. The Combustion Institute, Munich, Germany, 1986. 1917-1924

[3] Proust, C, Veyssiere, B. Fundamental Properties of Flame Propagating in Starch Dust-air Mixtures. Combustion Science and Technology, 1988, 62(4): 149-172

[4] Goroshin, S, Bidabadi, M, Lee, J H. Quenching Distance of Laminar Flame in Aluminum Dust Clouds. Combustion and Flame, 1996, 105(1/2): 147-160

[5] Eckhoff, R K. Dust Explosions in the Process Industries. 2nd ed.. Oxford: Butterworth-Heinemann, 1997

[6] Han, O-S, Yashima, M, Matsuda, T, et al. A Study of Flame Propagation Mechanisms in Lycopodium Dust Clouds Based on Dust Particles Behavior. Journal of Loss Prevention in the Process Industries, 2001, 14(3): $153-160$

[7] Dreizin, E L, Shoshin, Y L, Mudryy, R S, et al. Constant Pressure Flames of Aluminum and Aluminummagnesium Mechanical Alloy Aerosols in Microgravity. Combustion and Flame, 2002, 130(4): 381-385

[8] Eckhoff, R K. Prevention and Mitigation of Dust Explosions in the Process Industries: a Survey of Recent Research and Development. Journal of Loss Prevention in the Process Industries, 1996, 9(1): 3-20

[9] Hinze, J O. Turbulence. 2nd ed.. New York: McGrawHill, 1975

[10] Pu, Y K, Li, Y C, Kauffman, C W, et al. The Determination of Turbulence Parameters in Closed Explosion Vessels. AIAA Progress in Astronautics and Aeronautics, 1989, 132: 107-123

[11] $\mathrm{Pu}, \mathrm{Y} \mathrm{K}$, Jarosinski, J, Tai, C S, et al. The Investigation of the Feature of Dispersion-induced Turbulence and Its Effects on Dust Explosions in Closed Vessels. In: Proceedings of 22nd Symposium (International) on Combustion. The Combustion Institute, Seattle, USA, 1988. $1777-1787$

[12] Tamanini, F, Chaffee, J L. Turbulent Unvented Gas Explosions Under Dynamic Mixture Injection Conditions. In: Proceedings of 23rd Symposium (International) on Combustion. The Combustion Institute, Orléans, France, 1990. $851-858$

[13] Amyotte, P R, Chippett, S, Pegg, M J. Effects of Turbulence on Dust Explosions. Prog. Energy Combust. Sci., 1988, 14(4): 293-310 